\title{
Le concept d'espace dans le discours électronique et son rôle dans la construction des relations interpersonnelles
}

\author{
Verena THALER \\ Universität Mannheim \\ Romanisches Seminar \\ thaler@phil.uni-mannheim.de
}

Recibido: 30/10/2011

Aceptado: 18/09/2012

\begin{abstract}
Résumé
La présente contribution s'intéresse aux conversations électroniques françaises telles qu'elles se déroulent dans les salons de chat (ou tchatches) sur Internet. Elle étudie en particulier les mécanismes $\mathrm{du}$ travail relationnel employés dans ces conversations. Comment se construisent les relations interpersonnelles dans une conversation anonyme, purement textuelle et privée de tout contact physique entre les interlocuteurs ? Notre approche intègrera la théorie de la politesse de Brown et Levinson (1978, 1987) ainsi que le concept d'espace tel qu'il est conçu dans le contexte du discours électronique. Il s'avère que le recours à des espaces virtuels et fictifs joue un rôle important dans la construction des relations interpersonnelles, analysée ici en termes de stratégies de politesse positive.
\end{abstract}

Mots clés: chat, discours électronique, espace virtuel, face, politesse

\section{El concepto de espacio en el discurso electrónico y su papel en la construc- ción de las relaciones interpersonales}

\section{Resumen}

La presente contribución se centra en las conversaciones electrónicas francesas tal y como se desarrollan en los salones de chat de Internet, en particular, el estudio de los mecanismos del trabajo relacional empleados en estas conversaciones. ¿Cómo se construyen las relaciones interpersonales en una conversación anónima puramente textual y privada de todo contacto físico entre los interlocutores? La cuestión se aborda integrando la teoría de la cortesía de Brown y Levinson $(1978,1987)$ así como el concepto de espacio tal y como se concibe en el contexto del discurso electrónico. El recurso a espacios virtuales y ficticios parece desempeñar un importante papel en la construcción de las relaciones interpersonales, analizada aquí en términos de estrategias de cortesía positiva.

Palabras clave: chat, discurso electrónico, espacio virtual, face, cortesía 
The concept of space in electronic discourse and its role in the construction of interpersonal relations

\begin{abstract}
This paper is concerned with computer-mediated communication in French chat rooms. It focuses on mechanisms of relational work used in chat conversations. How do speaker and hearer construct interpersonal relations in anonymous, purely textual conversations, without any physical contact? This question is discussed within the framework of politeness theory (Brown \& Levinson 1978, 1987), and through an examination of the concept of space as it appears in electronic discourse. It turns out that virtual and fictitious spaces play an important role in the construction of interpersonal relations, which are analyzed in terms of positive politeness strategies in this paper.
\end{abstract}

Keywords: chat, electronic discourse, virtual space, face, politeness

\title{
Referencia normalizada
}

Thaler, V. (2013). "Le concept d'espace dans le discours électronique et son rôle dans la construction des relations interpersonnelles". Thélème, Vol. 28, 259-276.

\section{Introduction}

Le réseau Internet est non seulement le plus vaste espace mondial de communication et d'information, mais aussi un important espace relationnel. Parmi les nombreux services que propose Internet, la présente contribution s'intéresse particulièrement aux chats (ou tchatches), des espaces électroniques de conversation à caractère collectif dans lesquels on se retrouve pour discuter en temps réel avec d'autres participants, généralement anonymes et identifiés uniquement à travers leurs pseudonymes ${ }^{1}$. Il s'agit d'un type de discours qui, par son caractère spontané, éphémère et interactif, se rapproche de l'interaction spontanée en face à face. Dans sa réalisation concrète, le discours électronique a pourtant ses spécificités qui le distinguent clairement de l'interaction en face à face. La distance physique entre les interlocuteurs exclut tout contact visuel et auditif et, par conséquent, tout un nombre de moyens d'expression dont des éléments non-verbaux et paraverbaux. En outre, il faut noter que le système rend impossible toute sorte de réaction ou d'intervention de l'interlocuteur pendant la production du discours. Le message étant élaboré et reçu en bloc, il existe toujours un décalage entre la production du message et son apparition sur l'écran. Cette particularité, aussi peu significative qu'elle puisse paraître, a des répercussions importantes sur l'organisation du discours et certaines

${ }^{1}$ Pour plus de détails sur le fonctionnement des chats voir entre autres : Anis, 1999 et 2003 ; Pierozak, 2000a et 2003a. 
pratiques langagières des conversations électroniques ${ }^{2}$ (Thaler 2005). Il s'agit donc d'une forme de communication particulière, située entre l'oral et l'écrit ${ }^{3}$, avec tout un nombre de caractéristiques discursives et linguistiques propres à cette nouvelle forme d'interaction à travers Internet. Plusieurs travaux ont étudié les particularités linguistiques des chats français telles que différents types de graphies phonétisantes, variations et réductions morphosyntaxiques, l'emploi d'émoticônes (ou smileys), d'onomatopées et d'interjections, la réduplication de lettres ou de signes de ponctuation, une faible segmentation des messages, un grand nombre de constructions elliptiques et une forte présence d'anglicismes ainsi que de termes provenant du français familier, du français argotique ou du verlan (Anis, 1998, 1999 et 2003 ; Pierozak, 2000b, 2003a et 2003b ; Thaler, 2003 ; Krautgartner, 2003 ; Dejond, 2006 ; Van Compernolle \& Williams, 2007). En revanche, on a jusqu'à présent porté peu d'intérêt aux processus interactionnels en œuvre derrières ces formes linguistiques. Comment se construisent les relations interpersonnelles dans une forme de communication anonyme, purement textuelle, avec des moyens d'expression réduits, privée de tout contact physique entre les interlocuteurs? La présente contribution tentera de répondre à cette question à base d'un corpus de conversations électroniques françaises d'une durée de 6 heures et 15 minutes, soit 4.500 messages (ou 5.554 messages, les messages-systèmes compris). Ces conversations ont été enregistrées dans différents chats francophones ${ }^{4}$ pendant une période de 13 mois. Nous avons en particulier sélectionné des salons publics qui ne sont pas consacrés à un sujet de conversation spécifique et qui permettent par là même d'observer les mécanismes du travail relationnel. Dans les exemples cités ici, l'ensemble des pseudonymes et autres références personnelles permettant d'identifier les personnes concernées ont été anonymisés.

L'analyse proposée ici s'appuie sur le concept de face (Goffman, 1967) et son application dans la théorie de la politesse (Brown \& Levinson 1978 et 1987). Nous nous intéresserons en particulier aux stratégies de politesse positive qui jouent un rôle particulièrement important dans le travail relationnel des conversations électroniques (section 2). Dans la majorité des cas ces stratégies de politesse positive nécessitent un ancrage dans l'espace, sachant que, à la différence de l'interaction en face à face, les participants d'un chat ne disposent pas d'un espace physique commun dans lequel se déroule la conversation. Nous examinerons quels types d'espaces sont présents dans une conversation électronique (section 3) et dans quelle

\footnotetext{
${ }^{2}$ Les termes conversations électroniques, discours électronique et conversations des chats sont ici employés de façon synonyme.

${ }^{3}$ Le langage des chats a été décrit, entre autre, comme « écrit oralisé », « langue orale scriptée » ou «parlécrit »(Anis, 1999 : 74f).

${ }^{4}$ Concrètement il s'agit des chats accessibles sur les sites suivants: http://chat.free.fr; http://www.chat-land.org; http://www.discut.fr et http://www.chat-fr.fr.
} 
mesure le recours à ces espaces contribue à la construction des relations interpersonnelles dans les conversations des chats (section 4).

\section{Face et politesse}

Nous partons du principe que toute interaction sociale repose sur une volonté des interactants d'être respectés et de respecter l'autre. Cette volonté est exprimée à travers les notions de face et de territoire développées par Goffman (1967 et 1971) et intégrées plus tard dans la théorie de la politesse de Brown et Levinson (1978 et 1987). Brown et Levinson postulent que tout acteur social est investi d'une face positive et d'une face négative. La face positive (ou face chez Goffman) est la bonne image que nous voulons donner de nous-mêmes en public, le désir d'être respecté, reconnu et apprécié par les autres. La face négative (ou territoire chez Goffman) se réfère au désir de se préserver un espace personnel (corporel et social), une certaine sphère privée et liberté d'action. Tout individu est guidé par la volonté de préserver chacune des ces deux faces. Or, toute forme d'interaction sociale est potentiellement menaçante pour l'une des deux faces, d'où les fameux FTA (Face Threatening Act) de Brown et Levinson (1987: 61-62.) ${ }^{5}$. Par conséquent, l'interaction humaine est conditionnée par la considération des faces, appelée face work par Goffman, expression généralement traduite en français par travail de figuration $(1974: 15)^{6}$. Chacun cherche ainsi à faire respecter ses faces positive et négative et, inversement, à ménager les faces positive et négative de son interlocuteur, les relations humaines étant généralement réciproques. Dans la terminologie de Brown et Levinson ce travail de figuration se manifeste plus concrètement dans des stratégies de politesse, le terme de politesse étant employé non pas au sens courant du terme, mais en tant que concept théorique visant à expliquer certains mécanismes de l'interaction humaine. ${ }^{7}$ Dans cette perspective, la politesse est un moyen de concilier le désir mutuel de préserver les faces, avec le fait que la plupart des actes de langage sont potentiellement menaçants pour l'une des deux faces (Brown \& Levinson 1987). Les stratégies de politesse proposées par Brown et Levinson sont regroupées en cinq groupes de stratégies, numérotés par ordre de

\footnotetext{
${ }^{5}$ Le postulat de Brown et Levinson que pratiquement tous les actes sont des menaces potentielles aux faces a été critiqué comme vision trop pessimiste de l'interaction sociale (Schmidt, 1980 ; Lavandera, 1988 ; Kasper, 1990 ; Manno, 1998).

${ }^{6}$ Plus précisément, Goffman définit le travail de figuration (face work) comme «tout ce qu'entreprend une personne pour que ces actions ne fassent perdre la face à personne (y compris ellemême) » (1974:15).

${ }^{7}$ Pour la distinction entre un terme courant de politesse (first-order politeness) et un concept théorique de politesse (second-order politeness) voir Watts et al., 1992 ; Eelen, 1999 et 2001 ; Watts, 2003 ; Thaler, 2011.
} 
politesse croissante (voir Fig.1), chaque groupe comportant un certain nombre de sous-catégories.

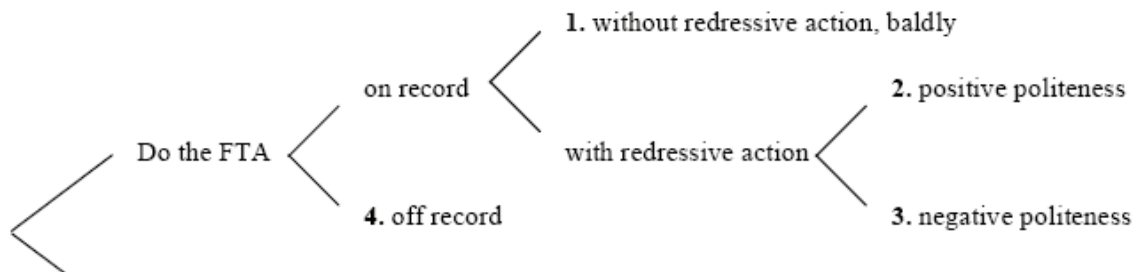

5. Don't do the FTA

Fig.1. Strategies de politesse (Brown \& Levinson 1987 : 60)

Sans discuter en détail ces stratégies et leurs caractéristiques respectives, nous allons nous concentrer dans ce qui suit sur la politesse positive qui joue un rôle particulièrement important dans les conversations étudiées ici. Dans le modèle de Brown et Levinson (1987: 102) la politesse positive comporte quinze sousstratégies ${ }^{8}$ :

01. Manifestez à A attention et prévenance

02. Exagérez votre approbation

03. Manifestez de l'intérêt envers l'interlocuteur

04. Utilisez des marqueurs d'identité « in-group »

05. Cherchez l'accord (sélection de thèmes consensuels)

06. Evitez le désaccord

07. Enoncez des lieux communs (référence au savoir partagé)

08. Faites des plaisanteries

09. Tenez compte des désirs de A

10. Faites des offres et promesses à $\mathrm{A}$

11. Soyez optimiste

12. Incluez à la fois $\mathrm{L}$ et $\mathrm{A}$ dans l'activité

13. Fournissez des raisons

14. Revendiquez la réciprocité

15. Faites à A des cadeaux (sous forme de biens, ou marques de sympathie, compréhension, coopération)

Toutes ces stratégies sont orientées vers la face positive de l'interlocuteur dans la mesure où elles visent à satisfaire le désir de l'interlocuteur d'être respecté et apprécié par l'autre'. Il s'avère que bon nombre de ces stratégies sont très fréquentes dans

\footnotetext{
${ }^{8}$ Pour la traduction française des stratégies voir Kerbrat-Orecchioni, $1992: 174$ et 175.

${ }^{9}$ Pour tenir compte des effets positifs et valorisants de telles stratégies sur la face, Kerbrat-
} 
les conversations électroniques des chats. Les processus relationnels qu'on observe dans ces conversations sont souvent liés à l'emploi de l'une ou l'autre de ces stratégies de politesse positive ou une combinaison de plusieurs stratégies dans un même message. Comme nous allons le voir dans les exemples discutés infra, le processus de s'adresser à un inconnu, d'entrer en contact avec lui, d'établir et de maintenir une relation amicale, ne serait-ce que pour la durée de la conversation, ne peut fonctionner qu'à travers un emploi massif de ces stratégies dans le contexte particulier des chats. A la différence de l'interaction en face à face, le travail relationnel dans les conversations électroniques est réalisé exclusivement au niveau textuel. Ce qui dans les conversations en face à face est souvent communiqué de façon non-verbale, par exemple par un regard, un sourire ou un geste, est compensé dans les conversations électroniques par un emploi massif de ces stratégies au niveau textuel. En outre, il s'avère que l'emploi de stratégies de politesse positive nécessite souvent un ancrage dans l'espace. Tout comme dans la communication en face à face, où les interactants disposent d'un espace commun de communication, les participants d'un chat cherchent à se créer - par leur discours - des espaces qui leur permettent de réaliser les stratégies en question. Dans ce qui suit, nous examinerons de quels types d'espaces il s'agit. Nous analyserons ensuite, à travers quelques exemples, dans quelle mesure le recours à ces espaces joue un rôle important dans la réalisation des stratégies de politesse positive.

\section{Le concept d'espace dans le discours électronique}

Les participants d'une conversation électronique se trouvent chacun devant son ordinateur pendant qu'ils discutent dans l'espace virtuel du chat. Cet espace virtuel existe en parallèle des espaces réels des participants sans être complètement indépendant d'eux. Regardé de près, nous observons une sorte d'interpénétration entre réel et virtuel (Schroer, 2003). Les interactants se trouvent à la fois dans l'espace virtuel du chat et dans leurs espaces réels respectifs, leurs activités dans les deux espaces pouvant se chevaucher. En outre, nous allons voir qu'à l'intérieur même de ces espaces peuvent se construire d'autres espaces (virtuels ou fictifs) au cours d'une conversation électronique. Le concept d'espace auquel nous avons recours en analysant la structure spatiale d'une conversation électronique n'est plus l'espace euclidien à trois dimensions, mais un concept relationnel de l'espace, plus adapté à la réalité des nouveaux médias de communication (Paetau, 1997 ; Schroer, 2003). Un espace virtuel comme un salon de chat n'a pas de dimensions physiques, ni une

Orecchioni (1992 et 2005) a introduit le terme de FFA (face flattering acts) par opposition aux FTA (face threatening acts). Dans cette terminologie, la politesse négative sert à éviter ou adoucir les FTA, tandis que la politesse positive produit principalement des FFA. 
existence permanente. Il se crée avec l'activité des utilisateurs et cesse d'exister quand les activités s'arrêtent.

Entre espaces réels et virtuels, reliés entre eux de différentes façons, quels sont les espaces qui apparaissent dans le discours électronique des chats? En analysant les conversations on constate qu'on y trouve au moins quatre types d'espace différents, à savoir (1) l'espace écran, (2) l'espace réel des participants, (3) l'espace virtuel du salon de conversation, et (4) des espaces fictifs (voir aussi Thaler 2012b).

\section{(1) Espace écran}

Pendant la durée de la conversation celle-ci s'affiche sur les écrans des participants. L'écran en tant qu'objet matériel permet la matérialisation du flux de données envoyé à partir d'un serveur vers les ordinateurs des utilisateurs connectés. Ce qu'on voit affiché à l'écran est perçu comme la réalisation matérielle de la conversation. L'écran devient ainsi un espace communicatif auquel les participants d'un chat peuvent faire référence dans leur conversation, comme dans les exemples (1) et $(2)^{10}$.

$$
\begin{aligned}
& <\text { damien> au faite, c'est quoi les Zzz... à coté de mon pseudo en haut? } \\
& <\text { Amos> regarde plus haut j'l'ai dis }
\end{aligned}
$$

Les expressions « en haut» (exemple 1) et «plus haut» (exemple 2) se réfèrent à la réalisation matérielle de la conversation sur l'écran, qu'il s'agisse d'un écran particulier (par exemple celui du producteur ou celui du destinataire du message en question) ou de l'ensemble de tous les écrans sur lesquels s'affiche simultanément la conversation.

\section{(2) Espace réel}

Dans les conversations on trouve également des références à l'entourage réel des participants, c'est à dire aux endroits où ils se trouvent au moment même où ils participent au chat (devant leurs ordinateurs, à la maison, au travail, dans un cybercafé, ou autre). Ces endroits font partie du contexte situationnel de la conversation dans la mesure où ils peuvent apparaître dans le discours et avoir une influence sur sa production.

$$
\begin{aligned}
& <\text { Amos }>\text { bon j'vais m'faire un chocolat chaud moi } \\
& <\text { Bounty }><\text { Amelie }>\text { au tel avec ma chérie j'allais pas te calinouner, elle m'a déjà fait une } \\
& \text { scène a cause de ça... }
\end{aligned}
$$

${ }^{10}$ Les exemples (1) et (2) ainsi que tous les exemples suivants sont tirés du corpus sur lequel se base l'étude. Dans les exemples, tout message est précédé du pseudonyme du producteur du message. Dans certains exemples, ce pseudonyme est suivi d'un deuxième pseudonyme qui est celui de la personne à laquelle s'adresse le message. 
Les messages des exemples (3) et (4) font référence à des activités que les participants exercent pendant qu'ils participent à la conversation (se faire un chocolat chaud (exemple 3) et parler au téléphone (exemple 4)). Ces activités influent sur la conversation en cours dans la mesure où elles expliquent une absence ou un manque d'activité du participant concerné. Il n'y a pas de référence explicite à l'espace en question, mais les messages évoquent nécessairement une image de l'entourage de l'interlocuteur dans lequel se situe l'activité. Plus exactement ce n'est donc pas l'espace réel du locuteur qui est intéressant dans l'interaction, mais l'idée d'un tel espace que se fait son interlocuteur à partir du message reçu.

\title{
(3) Espace salon de conversation
}

La conversation électronique se déroule dans ce qu'on appelle un « salon de conversation » (que certains appellent aussi « chambre » ou « pièce (de conversation) », cf. Pierozak, 2000b). L'idée d'un salon virtuel dans lequel on se retrouve pour discuter donne un cadre à la conversation électronique et la rapproche d'une conversation en face à face. Ce cadre sert de repère et crée un espace commun de communication.

$$
\begin{aligned}
& <\text { Lolo01> ya des fille sur ce salon?? } \\
& <\text { Mika }>\text { Salut claude, bienvenue dans cette salle } \\
& <\text { moon2008> c la premiere fois que je parle ici } \\
& <\text { wizix }>\text { marianne t es la? }
\end{aligned}
$$

Certaines situations peuvent nécessiter un recours explicite au salon de conversation, comme dans les exemples (5) et (6) (" sur ce salon », " dans cette salle »). Dans d'autres cas on y fait référence par un adverbe (ici (exemple 7), là (exemple $8)$ ). Dans de nombreux cas on trouve aussi des références implicites à l'espace commun de communication, par exemple en évoquant le contact visuel ( $«$ je te vois », « je suis content de te voir », etc.) ou le contact physique entre les interlocuteurs (p.e. « Bounty prend Amelie dans ses bras et lui fait de gros calinous »).

\section{(4) Espaces fictifs}

Au cours d'une conversation électronique les participants font également référence à des espaces fictifs. Ils créent, à travers leur discours, des endroits imaginaires qui leur permettent la mise en scène d'activités fictives. La plupart du temps ils s'imaginent eux-même dans ces espaces. Ils élargissent ainsi leur rayon d'action et créent des situations pour des activités communes. Max vas sasoir pres de Ptite_Melanie, la prend dans ces bras et dit : "mais arete de pleurer"

\author{
Amos attrape Lisa dans ses bras \\ Lisa donne un coup de pieds à Amos et se cache derrere Jeff :\$
}


$<$ tristan $>$ Cherche PV !!!

Ludo met sa casquette de gendarme et colle un PV a tristan de $135 €$ pour stationnement sur place handicapée

Les exemples (9)-(11) contiennent des messages à la troisième personne, ce qui est une façon de décrire des actions dans les conversations électroniques. Le producteur du message parle de lui-même à la troisième personne et implique généralement d'autres personnes dans ses actions fictives. Très souvent il s'agit d'actions qui permettent de simuler un contact physique avec l'interlocuteur, comme dans les exemples (9) et (10). Dans l'exemple (9), Max s'approche physiquement de son interlocutrice en s'assoyant près d'elle et en la prenant dans ses bras. L'exemple (10) représente une activité ludique, Amos voulant attraper son interlocutrice Lisa et celle-ci lui donnant un coup de pied et se cachant derrière un autre participant. Les deux exemples pourraient soit se situer dans l'espace même du salon de conversation (si on s'imagine que c'est un salon dans lequel on peut se déplacer et s'asseoir l'un à côté de l'autre) soit dans un espace fictif, indépendamment du contexte virtuel du salon de conversation. L'exemple (11) se situe clairement dans un espace imaginaire en dehors du salon de conversation. L'énoncé «Cherche PV », signifiant « Je cherche quelqu'un pour une conversation en privé $\gg{ }^{11}$ est repris par son interlocuteur dans un jeu de mots. La signification de PV a changé, passant de «privé » à « procès verbal ». Pour contextualiser le jeu de mots, Ludo construit une situation fictive qui est celle d'une voiture mal garée et d'un gendarme qui lui donne une amende (« Ludo met sa casquette de gendarme et colle un PV a tristan de $135 €$ pour stationnement sur place handicapée »).

On constate que, dans une conception relationnelle de l'espace, il n'est pas toujours facile de délimiter les différents espaces en jeu dans une conversation électronique. Les participants créent des espaces à travers leur discours et les mettent en relation entre eux, toujours en fonction des besoins discursifs de la situation.

Dans une approche de sémantique cognitive, la création d'espaces pourrait aussi être décrite à l'aide du concept d'espace mental. Selon la théorie des espaces mentaux (Fauconnier, 1985 et 1997), différents espaces se créent pendant le discours. Ces espaces peuvent se référer à des entités réelles mais aussi virtuelles et sont généralement reliés entre eux, permettant de passer d'un espace à l'autre au fil du discours. Dans cette conception, différentes formes linguistiques employées dans une conversation électronique (comme celles employées dans les exemples (1)-(11)) évoqueraient différents types d'espaces mentaux représentant des situations réelles, virtuelles ou fictives.

${ }^{11}$ Outre les salons publics, accessibles à tout le monde, les systèmes de chats permettent aussi la création de salons privés qui sont souvent utilisés pour des conversations à deux. 
Dans ce qui suit, nous nous intéresserons moins aux bases théoriques d'une construction d'espaces qu'à la question de savoir quel rôle la construction de tels espaces peut jouer dans la réalisation des stratégies de politesse positive. Cette question sera étudiée à travers quelques extraits de conversations électroniques.

\section{Espace et politesse positive dans le discours électronique}

Parmi les quatre types d'espaces considérés supra, ce sont en particulier les deux derniers, à savoir l'espace virtuel du salon de conversation et les espaces fictifs, qui sont très présents dans les conversations électroniques. Ils permettent aux participants de dépasser les limites physiques de la conversation médiatisée par ordinateur et de simuler des situations de contact, conformément à leur volonté de faire des rencontres et de nouer des relations avec les interlocuteurs. Le recours à un espace virtuel ou fictif peut en effet servir de base pour la construction de relations interpersonnelles dans le chat. Ce recours s'avère souvent un moyen très efficace pour la mise en œuvre de stratégies de politesse positive, destinées à la valorisation de la face positive de l'interlocuteur (Thaler 2012b). Par la suite, nous nous intéresserons de plus près à ce processus de travail relationnel. Nous choisirons, en guise d'exemples, quelques stratégies particulièrement fréquentes pour illustrer le lien entre la construction d'espaces et l'emploi de stratégies de politesse ${ }^{12}$.

\subsection{Gestes de sympathie et de complicité}

Dans l'interaction en face à face un rapprochement social est souvent lié à un rapprochement physique. La distance entre les interlocuteurs a tendance à se réduire. On se rapproche l'un de l'autre, on se regarde dans les yeux, on échange des gestes de complicité. Dans les conversations électroniques on emploie des stratégies qui reproduisent, de façon verbale ou symbolique, ce rapprochement physique entre les interlocuteurs. Ce rapprochement peut, entre autres, être exprimé à travers des gestes de sympathie et de complicité, c'est à dire des imitations textuelles de gestes ou d'expressions mimiques tel que serrer la main à quelqu'un, prendre quelqu'un dans ses bras, faire une bise à quelqu'un ou sourire à quelqu'un. Ces gestes, réalisés de façon verbale, peuvent être analysés en tant que stratégies de politesse positive, visant à valoriser la face positive de l'interlocuteur. Concrètement, peuvent être concernées, en fonction du contexte, la stratégie 1 (Manifestez attention et prévenance à A), la stratégie 3 (Manifestez de l'intérêt envers l'interlocuteur) ou la stratégie 15 (Faites des cadeaux à $\mathrm{A}$ ). Des gestes de sympathie sont souvent des cadeaux symboliques qu'on fait à l'interlocuteur. Ce sont en effet des stratégies très fréquentes dans les conversations électroniques qui jouent un rôle important dans la cons-

\footnotetext{
${ }^{12}$ Pour d'autres stratégies de politesse employées dans les conversations électroniques voir Thaler 2012a.
} 
truction des relations interpersonnelles. Les extraits (12)-(15) en donnent quelques exemples.

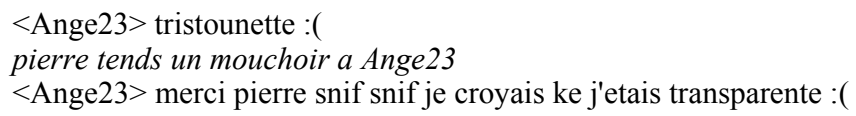

$<$ Ange23> tristounette :( pierre tends un mouchoir a Ange 23

$<$ Ange23 $>$ merci pierre snif snif je croyais ke j'etais transparente :(

Kevin sert la main à son pote fumika

Marco s'endors sur les genoux de Samia

Samia gratouille la tete de Marco

$<$ Lolo $><$ Amelie $>$ tu nous quitte ? Pardon, tu me quitte?

$<$ Amelie $><$ Lolo $>$ je sais pas encore

$<$ Lolo $><$ Amelie $>$ nan reste !

Lolo sers Amelie contre lui

L'exemple (12) représente une prise de contact entre deux participants à travers un geste d'attention simulé. Ange23, jusque là peu active dans la conversation, se montre triste. En réaction, Pierre manifeste son attention (stratégie 1) et son intérêt envers elle (stratégie 3) en lui tendant un mouchoir (stratégie 15) et fait ainsi appel à sa face positive. La réaction de l'interlocutrice montre qu'elle recherchait effectivement l'attention de l'autre ( « merci pierre snif snif je croyais ke j'etais transparente ») et que le geste d'attention de Pierre a stimulé sa face positive. Dans la suite de la conversation (qui n'est pas reproduite ici) on voit que la simulation de ce geste a permis de créer un lien entre les deux interlocuteurs et de les rapprocher dans leur relation. Un effet similaire peut être observé dans les exemples (13), (14) et (15), qu'il s'agisse d'un geste plutôt formel comme serrer la main à l'interlocuteur (exemple 13) ou de gestes plus intimes comme se reposer sur les genoux de l'autre, gratouiller sa tête (exemple 14) ou le serrer dans ses bras (exemple 15). Il est intéressant à observer que ces gestes affectifs sont souvent liés à l'emploi de la troisième personne. Cette dernière semble entre autre avoir une fonction de prise de distance face aux actes décrits. Le producteur du message parle de lui-même à la troisième personne pour indiquer que ce n'est pas son intention de menacer la face négative de l'interlocuteur. C'est par ce biais qu'un acte potentiellement menaçant peut se transformer, dans le contexte donné, en stratégie de politesse positive.

Ce qui joue un rôle particulièrement important dans ce contexte, c'est l'emploi d'émoticônes ou smileys. Ce sont des combinaisons de caractères typographiques qui sont censées représenter des émotions ou expressions faciales telles qu'un sourire (voir lignes 2, 3 et 8 de l'exemple (16)), un clin d'œil (ligne 3), une bise, représentées ici par (K) pour kiss (lignes 4 et 5), ou une expression gênée ou timide 
(ligne 7). ${ }^{13} \mathrm{Vu}$ leur forme réduite, les émoticônes sont un moyen particulièrement économique d'exprimer un geste de sympathie envers l'autre. ${ }^{14}$

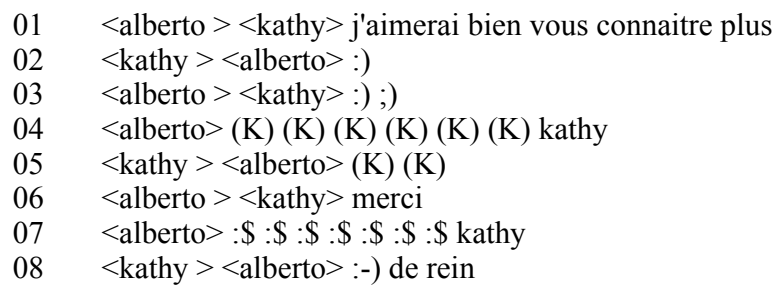

Tous ces gestes impliquent la coprésence des interlocuteurs. Ils ne peuvent fonctionner que dans un espace commun qui est l'espace virtuel du salon de conversation. Cet espace virtuel sert de toile de fond à ces gestes fictifs. La mise en scène verbale de tels gestes de sympathie, voire de tendresse, est souvent une façon de jouer avec l'identité virtuelle et la liberté de dépasser les normes et les contraintes de la vie réelle. Mise à part cette fonction ludique, ils ont aussi une fonction discursive importante, dans la mesure où ils constituent un élément important du travail de figuration et contribuent ainsi au fonctionnement de l'interaction dans le chat.

\subsection{Activités fictives}

Au-delà d'un simple geste, on trouve aussi des activités plus complexes, des interactions fictives que les participants du chat mettent en place. Un moyen de faciliter un rapprochement social est de simuler une activité partagée qui est typiquement associée à une relation proche. Dans le corpus analysé on trouve toute une gamme d'activités de ce genre, telles que trinquer, boire de l'alcool, danser, offrir à boire ou à manger à l'autre, écouter de la musique, caresser l'autre ou lui faire un massage, s'appuyer sur lui, le consoler, le protéger, courir après lui et essayer de l'attraper, etc. En incluant l'autre dans une activité ou en l'invitant à faire une activité partagée on lui fait comprendre qu'on s'intéresse à lui, qu'on cherche à entrer en contact ou à approfondir le contact avec lui. C'est donc une stratégie qui fait appel à la face positive de l'interlocuteur, même s'il s'agit d'une activité qui, au premier abord, semble peu respectueux, comme dans l'exemple (17).

01 Jeff21 ecrase Lisa, il a pa vu kel étai sou lui

$02<$ Lisa $><$ Jeff21 $>$ 'culay :'(

${ }^{13}$ Dans la plupart des chats actuels les émoticônes peuvent être insérés sous forme de petites images.

${ }^{14}$ Dans leur étude sur le rôle des smileys dans le discours électronique Marcoccia et Gauducheau (2007) montrent comment l'emploi de smileys peut servir de marqueur de proximité. Il permet à l'auteur d'un message d'indiquer qu'il entretient ou aimerait entretenir une relation de familiarité ou de connivence avec son destinataire (voir aussi Marcoccia, 2000). Dans d'autres cas, les smileys peuvent aussi servir à désamorcer le caractère menaçant ou agressif d'un énoncé (voir exemple 17). 
$03<$ Jeff21 $>$ sapercevant prefere caliner Lisa

$04<$ Lisa $><$ Jeff $21>$ jprefere $: \$$

$05<$ Jeff21 $><$ Lisa $>$ :x:x:x:X

$06<$ Lisa $><$ Jeff2 $1>=$ )

Malgré le caractère agressif du geste ( $«$ Jeff 21 ecrase Lisa, il a pa vu kel étai sou lui »), nous avons affaire à une stratégie de politesse positive, l'intention étant de manifester de l'attention (stratégie 1) et de l'intérêt envers l'autre (stratégie 3) et de l'inclure dans une activité (stratégie 12) pour se rapprocher de lui. La scène imaginée par Jeff2 1 se situe dans un espace fictif, voire dans une dimension fictive. Il est difficile d'imaginer, dans des dimensions réelles, une situation dans laquelle une personne écraserait une autre parce qu'elle n'aurait pas vu qu'elle était sous elle. Le producteur du message joue avec cette dimension fictive qu'il crée à travers son énoncé et qui sert sans doute à attirer l'attention de l'interlocutrice impliquée dans son message. C'est le caractère fictif qui fait que le geste d'écraser l'interlocutrice n'est pas perçu comme un geste réellement agressif. Il s'agit, en toute apparence, d'une activité ludique qui est tellement loin de la réalité qu'elle n'est pas menaçante pour la face de l'interlocutrice, mais sert, au contraire, de moyen pour se rapprocher d'elle.

Dans l'extrait (18) une stratégie de politesse positive est développée à partir d'un espace réel qui est mis en relation avec une activité imaginée par l'un des participants à la conversation.

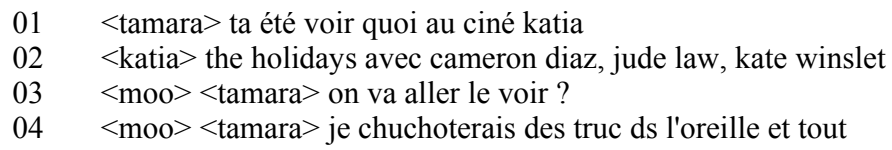

La question en ligne 1 fait référence à un espace réel et à une activité réelle dans le passé (Katia est allée voir un film au cinéma). En ligne 3 et 4, Moo reprend cet élément réel et l'utilise pour construire une situation imaginaire qui est celle d'aller au cinéma avec son interlocutrice et de se retrouver dans une situation d'intimité avec elle (« je chuchoterais des truc ds l'oreille et tout»). Il s'agit d'un message qui peut être attribué à plusieurs stratégies de politesse positive, à savoir la stratégie 1 (Manifestez attention et prévenance à $\mathrm{A}$ ), la stratégie 3 (Manifestez de l'intérêt envers l'interlocuteur), la stratégie 10 (Faites des offres et promesses à A) et la stratégie 12 (Incluez à la fois $\mathrm{L}$ et $\mathrm{A}$ dans l'activité). Dans une situation en face à face une telle invitation pourrait être menaçante pour la face négative de l'interlocutrice. La façon dont est présentée l'invitation ne serait pas forcément appropriée en face à face envers une personne qu'on connaît à peine. Dans la conversation de chat il ne s'agit pas tellement d'une invitation réelle au cinéma, mais plutôt d'une stratégie verbale employée par Moo dans le but de faire comprendre à son interlocutrice qu'il s'intéresse à elle et qu'il cherche à créer un lien amical avec elle. La volonté de valoriser la face positive de l'interlocutrice est liée à la volonté d'établir une relation amicale avec elle. 


\subsection{Construction d'activités fictives à partir de pseudonymes}

Les pseudonymes que choisissent les participants ${ }^{15}$ peuvent devenir des éléments discursifs dans la mesure où ceux-ci peuvent déclencher des interactions liées à ces pseudonymes. Nous nous intéressons ici en particulier aux cas où un pseudonyme sert de point de départ pour la réalisation de stratégies de politesse positive.

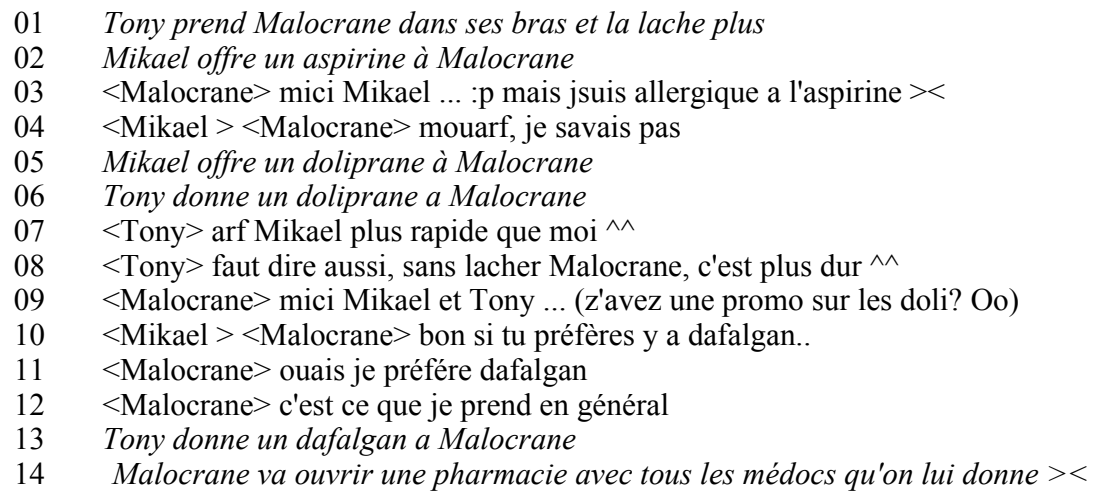

Dans l'exemple (19) l'une des participantes s'appelle Malocrane ('mal au crâne'), un pseudonyme qui provoque toute une série de réactions. L'extrait commence par un geste de compassion et de consolation (« Tony prend Malocrane dans ses bras et la lache plus »), suivi d'un cadeau imaginaire sous forme d'un comprimé antidouleur ("Mikael offre un aspirine à Malocrane »). Les deux gestes sont clairement adressés à la face positive de l'interlocutrice et lui donnent l'occasion de réagir de son côté. Elle remercie Mikael et poursuit la conversation en disant qu'elle est allergique à l'aspirine (ligne 3). Ceci suscite d'autres réactions de la part des interlocuteurs qui lui offrent un doliprane (lignes 5 et 6), puis un dafalgan (ligne 13). Il se crée un discours amical autour de cet échange de médicaments qui implique des informations sur la vie réelle de la personne concernée ( jsuis allergique a l'aspirine », « ouais je préfére dafalgan », « c'est ce que je prend en général»), des réactions à ces informations ( « je savais pas », «bon si tu préfères y a dafalgan »), un meta-discours ludique autour de l'activité d'offrir des médicaments à l'interlocutrice (" arf Mikael plus rapide que moi $\wedge^{\wedge}$ », " faut dire aussi, sans lacher Malocrane, c'est plus dur $\wedge^{\wedge} \nu^{16}$ ) ainsi qu'un discours ludique faisant allusion à la quantité des médicaments en jeu ( « z'avez une promo sur les doli? », « Malocrane va ouvrir une pharmacie avec tous les médocs qu'on lui donne »). Si on essaie d'analyser la structure spatiale de cette interaction, on constate qu'il y a non seulement l'espace virtuel

\footnotetext{
${ }^{15}$ Dans la plupart des salons de chat, les participants ont la possibilité de changer leur pseudonyme au cours de la conversation.

${ }^{16}$ L'émoticône $^{\wedge}$ représente l'expression des yeux ou des sourcils d'une personne qui rit. Il donne ici un caractère plaisant aux messages qu'il accompagne.
} 
du salon de conversation qui est en jeu, mais aussi un espace fictif ( Malocrane va ouvrir une pharmacie avec tous les médocs qu'on lui donne ») et des éléments d'un espace réel. Ce n'est plus un simple échange d'informations ou de gestes d'attention, mais une interaction complexe qui intégre différents niveaux de discours, y compris des activités ludiques et plaisantes. L'enchaînement de ces activités crée une ambiance de complicité entre les trois personnes et comporte tout un nombre de stratégies de politesse positive, telles que la stratégie 1 (Manifestez attention et prévenance à A), la stratégie 3 (Manifestez de l'intérêt envers l'interlocuteur), la stratégie 9 (Tenez compte des désirs de $\mathrm{A}$ ), la stratégie 12 (Incluez à la fois $\mathrm{L}$ et $\mathrm{A}$ dans l'activité), la stratégie 15 (Faites des cadeaux à A) ou encore la stratégie 8 (Faites des plaisanteries). Cette dernière se fonde sur le fait que l'acte de plaisanter demande un certain degré de complicité et de confiance entre les interlocuteurs et contribue, en règle générale, à renforcer cette complicité et confiance.

Nous retrouvons des processus similaires dans l'exemple (20) qui joue, cette fois-ci, avec deux pseudonymes différents, à savoir sandrinette triste et Sadly'Me. Les deux pseudonymes suscitent des réactions affectives tout au long de l'extrait.

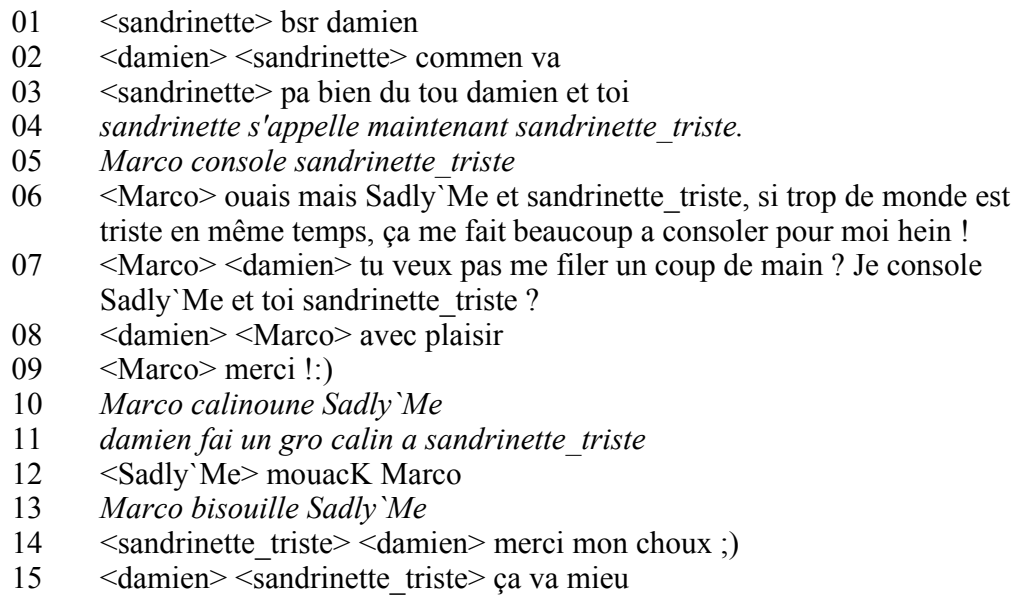

Les deux participants masculins, Marco et Damien, simulent, à travers leur discours, un espace fictif qui leur permet de consoler (lignes 6 et 7), "calinouner » (ligne 10), "bisouiller» (ligne 13) les interlocutrices et de leur faire des câlins (ligne 11). Les interlocutrices répondent par des remerciements (lignes 9 et 14), des bises (ligne 8), l'emploi d'un terme d'adresse hypocoristique ( mon choux », ligne 10 ), et, à la fin de l'extrait, la remarque que « ça va mieu[x]» (ligne 15). Toutes ces activités peuvent être analysées sous forme de stratégies de politesse, toutes destinées à valoriser la face positive de l'autre. Il s'agit, de toute évidence, de gestes affectifs exagérés destinés à simuler une relation proche et une ambiance de complicité entre les interlocuteurs. Nous observons, une fois de plus, à quel point la simulation d'activités fictives partagées, construites à partir de pseudonymes dans 
les exemples (19) et (20), peut contribuer au développement des relations interpersonnelles.

\section{Conclusion}

Nous avons vu que le recours à l'espace virtuel du salon de conversation ou à un espace fictif permet aux participants d'une conversation de chat de mettre en scène des gestes fictifs et des activités fictives. Ces gestes et ces activités servent, en grande partie, à valoriser la face positive de l'interlocuteur et peuvent ainsi être analysés sous forme de stratégies de politesse positive. L'emploi de ces stratégies correspond à la volonté des participants d'établir des contacts et de se rapprocher de l'autre. On constate que le rapprochement social est souvent lié à un rapprochement physique entre les interlocuteurs. Les stratégies de politesse positive se manifestent, entre autres, à travers des imitations textuelles de gestes de sympathie et de complicité ou d'activités fictives, toutes nécessitant un rapprochement dans l'espace. Suite au manque de contact physique entre les interlocuteurs et au manque de moyens d'expression non-verbaux et paraverbaux, ces stratégies se manifestent exclusivement au niveau textuel. C'est au niveau textuel que se crée un espace commun de communication à travers l'espace virtuel du salon de conversation ou des espaces fictifs. Dans cette perspective, on peut dire que le recours à ces espaces joue un rôle important non seulement pour la construction des relations interpersonnelles, mais aussi pour le fonctionnement des conversations électroniques tout court.

\section{RÉFÉRENCES BIBLIOGRAPHIQUES}

Anis, J., (1998) Texte et ordinateur. L'écriture réinventée? Bruxelles, De Boeck.

Anis, J., (1999) "Chats et usages graphiques" in J. Anis (éd.), Internet, communication et langue française. Paris, Hermès, pp. 71-90.

Anis, J., (2003) "Communication électronique scripturale et formes langagières: chats et SMS" in Actes des Quatrièmes Rencontres Réseaux Humains/Réseaux Technologiques [En ligne]. Poitiers, Université de Poitiers, disponible sur: $\mathrm{http}: / /$ rhrt.edel.univ-poitiers.fr/document.php?id=547 [Dernier accès le 30 octobre 2011].

Brown, P. \& S. C. Levinson, (1978) "Universals in language usage: Politeness phenomena" in Goody, E. N. (éd.), Questions and Politeness. Cambridge, Cambridge University Press, pp. 56-289.

Brown, P. \& S. C. Levinson, (1987) Politeness: Some universals in language usage. Cambridge, Cambridge University Press.

Dejond, A., (2006) Cyberlangage. Bruxelles, Éditions Racine.

Eelen, G., (1999) "Politeness and Ideology: A Critical Review" in Pragmatics. $\mathrm{N}^{\circ}$ 9/1, pp. 163-173.

Eelen, G., (2001) A Critique of Politeness Theories. Manchester, St. Jerome. 
Fauconnier, G., (1985) Mental Spaces: Aspects of Meaning Construction in Natural Language. Cambridge, MIT Press.

Fauconnier, G., (1997) Mappings in Thought and Language. Cambridge, Cambridge University Press.

Goffman, E., (1967) Interaction ritual: Essays on face-to-face behaviour. New York, Doubleday.

Goffman, E., (1971) Relations in public: microstudies of the public order. New York, Basic Books.

Kasper, G., (1990) "Linguistic politeness: Current Research Issues" in Journal of Pragmatics. $\mathrm{N}^{\mathrm{o}} 14 / 2$, pp. 193-218.

Kerbrat-Orecchioni, C., (1992) Les interactions verbales, t. II. Paris, Armand Colin.

Kerbrat-Orecchioni, C., (2005) Le discours en interaction. Paris, Armand Colin.

Krautgartner, K., (2003) "Techniques d'abréviation dans les webchats francophones" in Linguistik online [En ligne]. $\mathrm{N}^{0}$ 15, 3, disponible sur : http://www.linguistik-online.de/15_03/krautgartner.html [Dernier accès le 30 octobre 2011].

Lavandera, B., (1988) "The social pragmatics of politeness forms" in Ammon, U. et al. (éds.), Sociolinguistics/Soziolinguistik: An International Handbook of the Science of Language and Society/Ein internationals Handbuch zur Wissenschaft von Sprache und Gesellschaft, Vol. 2. Berlin/New York, De Gruyter, pp. 11961205.

Manno, G., (1998) "Politesse et typologie des textes" in Caron, B. (éd.), Actes du XVIe Congrès International des Linguistes. CD Rom. Oxford, Elsevier Sciences.

Marcoccia, M., (2000) "Les smileys: une représentation iconique des émotions dans la communication médiatisée par ordinateur" in Platin, C. et al. (éds.), Les émotions dans les interactions. Lyon, Presses universitaires de Lyon, pp. 249263.

Marcoccia, M., Gauducheau, N. (2007): "L'analyse du rôle des smileys en production et en réception : un retour sur la question de l'oralité des écrits numériques", in Glottopol. $\mathrm{N}^{\mathrm{o}} 10, \mathrm{pp} .39-55$.

Paetau, M. (1997): "Sozialität in virtuellen Räumen?", in B. Becker, M. Paetau (éds.), Virtualisierung des Sozialen. Die Informationsgesellschaft zwischen Fragmentierung und Globalisierung, Campus, Frankfurt/Main, pp. 103-134.

Pierozak, I. (2000a): "Approche sociolinguistique des pratiques discursives en français sur internet: "ge fé dais fotes si je voeux "”, in Revue Française de Linguistique Appliquée. Volume V, fascicule 1, pp. 89-104.

Pierozak, I. (2000b): "Les pratiques discursives des internautes en français : matériaux et éléments de réflexion." in Le Français Moderne. $\mathrm{N}^{\mathrm{0}}$ 68/1, pp. 109-129.

Pierozak, I. (2003a): Le français tchaté. Une étude en trois dimensions - sociolinguistique, syntaxique et graphique - d'usages IRC, 3 volumes, Université d'AixMarseille I, Aix en Provence, disponible sur : http://www.upicardie.fr/LESCLaP/spip.php?article 74 [Dernier accès le 17 octobre 2011]

Pierozak, I. (2003b): "Le « français tchaté » : un objet à géométrie variable ?" in Langage \& Société. N 104, pp. 123-144. 
Schmidt, R. W. (1980): "Review of Esther Goody (ed.), Questions and Politeness: Strategies in Social Interaction", in Regional English Language Centre Journal. $\mathrm{N}^{\mathrm{o}}$ 11, pp. 100-114.

Schroer, M. (2003): "Raumgrenzen in Bewegung. Zur Interpenetration realer und virtueller Räume", in Sociologia internationalis. $\mathrm{N}^{\mathrm{o}} 41$, pp. 55-76.

Thaler, V. (2003): Chat-Kommunikation im Spannungsfeld zwischen Oralität und Literalität, Verlag für Wissenschaft und Forschung, Berlin.

Thaler, V. (2005): "Zur Problematik der Synchronizität computervermittelter Kommunikation", in Zeitschrift für Angewandte Linguistik. N ${ }^{\circ}$ 43, Peter Lang, Frankfurt/Main, pp. 76-98.

Thaler, V. (2011): "La politesse verbale: Analyse d'un concept", in G. Held, U. Helfrich (éds.): Cortesia - Politesse - Cortesía. La cortesia verbale nella prospettiva romanistica. La politesse verbale dans une perspective romaniste. La cortesía verbal desde la perspectiva romanística. Aspetti teorici e applicazioni / Aspects théoriques et applications / Aspectos teóricos y aplicaciones, Peter Lang, Frankfurt/Main et al., pp. 59-74.

Thaler, V. (2012a): Sprachliche Höflichkeit in computervermittelter Kommunikation, Stauffenburg, Tübingen.

Thaler, V. (2012b): "Zur Bedeutung realer und virtueller Räume in beziehungsorientierter Online-Kommunikation", in: A. Gerstenberg, C. PolzinHaumann, D. Osthus (éds.): Sprache und Öffentlichkeit in realen und virtuellen Räumen. Akten der Sektion auf dem 7. Kongress des Frankoromanistenverbands (Essen, 29.9.-2.10.2010), Romanistischer Verlag, Bonn, pp. 197-216.

Van Compernolle, R. A., Williams, L. (2007): "De l'oral à l'électronique : la variation orthographique comme ressource sociolstylistique et pragmatique dans le français électronique", in Glottopol. $\mathrm{N}^{\mathrm{o}}$ 10, pp. 56-69.

Watts, R. J. (2003): Politeness, Cambridge University Press, Cambridge.

Watts, R. J., ide, S., ehlich, K. (1992): "Introduction", in R. J. Watts, S. Ide, K. Ehlich (éds.): Politeness in Language. Studies in its History, Theory and Practice. Mouton de Gruyter, Berlin, pp. 1-17. 